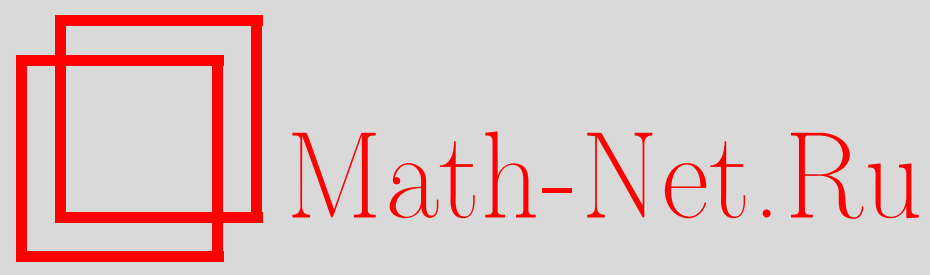

В. Т. Филиппов, Стандартные обертывающие коммутативных тройных систем, Матем. заметки, 2001, том 69, выпуск 5, 733-739

DOI: https://doi.org/10.4213/mzm536

Использование Общероссийского математического портала Math-Net.Ru подразумевает, что вы прочитали и согласны с пользовательским соглашением http://www . mathnet.ru/rus/agreement

Параметры загрузки:

IP : 54.237 .59 .107

26 апреля 2023 г., 02:36:25

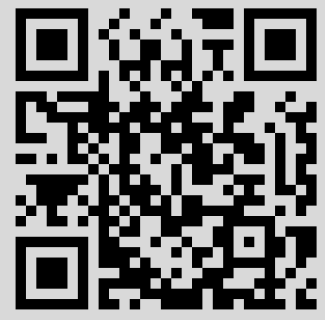


УДК 512.554

\section{СТАНДАРТНЫЕ ОБЕРТЫВАЮЩИЕ КОММУТАТИВНЫХ ТРОЙНЫХ СИСТЕМ}

\section{В. Т. Филиппов}

При некоторых ограничениях на характеристику поля $\Phi$ определяется коммутативная стандартная обертывающая $q$-алгебра $B$ коммутативной тройной системы $A$ над $\Phi$ и доказывается, что

1) если алгебра $B$ проста, то система $A$ проста;

2) если система $A$ проста, то $B$ либо проста, либо разлагается в прямую сумму (как идеалов) двух изоморфных простых подалгебр.

Библиограбия: 3 названия.

Тройной системой над полем $\Phi$ называется линейное пространство над $\Phi$ с трилинейной операцией.

В [1] показано, что произвольные коммутативная и антикоммутативная тройные системы над полем $\Phi$ характеристики $p \neq 3$ могут быть вложены соответственно в некоторые коммутативную и антикоммутативную (линейные) алгебры так, чтобы заданная на тройной системе операция $[x, y, z]$ совпадала с якобианом

$$
J(x, y, z)=(x y) z+(z x) y+(y z) x
$$

определенным в соответствующей алгебре (которая в [1] называлась стандартной $\beta$ обертывающей алгеброй системы). В коммутативном случаев эту алгебру вкладывается и йорданова алгебра правых умножений системы, т.е. алгебра, порожденная всеми правыми умножениями системы относительно операции $X \circ Y=X Y+Y X$.

Если $p \neq 2$, то в теории йордановьх алгебр чаще используется операция

$$
X \circ Y=\frac{1}{2}(X Y+Y X)
$$

(см., например, [2]). Чтобы охватить и этот случай, в настоящей работе для произвольной коммутативной системы $A$ вводится понятие стандартной обертьвающей $q$-алгебры $B$, где пространство алгебры $B$ является прямой суммой пространства системы $A$ и пространства йордановой алгебры $R^{\langle+\rangle}(A)$, порожденной всеми правьпи умножениями системы $A$ относительно операции

$$
X \circ Y=q(X Y+Y X),
$$

Работа выполнена при финансовой поддержке Российского фонда фундаментальных исследований, грант № 99-01-00499. 
a $q$ - произвольньй ненулевой элемент $\Phi$. При этом сохраняется связь между трилинейной операцией системы $A$ и якобианом ее стандартной обертывающей.

В [1] доказано, что простота антикоммутативной тройной системы равносильна простоте ее стандартной $\beta$-обертьвающей алгебры. В настоящей работе показьвается, что если $p \neq 2,3$, то с одной стороны, простота коммутативной тройной системы вытекает из простоты ее стандартной обертывающей $q$-алгебры $B$, а с другой, если система $A$ проста, то $B$ либо является простой, либо разлагается в прямую сумму двух изоморфных идеалов, которые являются простыми алгебрами.

Заметим, что результаты данной работы аналогичны результатам о стандартных обертывающих простых лиевых тройных систем (см., например, [3]).

Тройная система над полем $\Phi$ называется коммутативной, если в ней вьполняются тождества

$$
[x, y, z]=[y, x, z], \quad[x, y, z]=[x, z, y] .
$$

Отсюда следует, что операция $[x, y, z]$ симметрична относительно всех перестановок $x, y, z$.

Пусть $A$ - коммутативная тройная система над полем $\Phi$ с трилинейной операцией $[x, y, z], E$-алгебра эндоморфизмов пространства $A$. Эндоморфизм $R(y, z): x \rightarrow[x, y, z]$ назьвается правым умножением системы $A$, определенным элементами $y, z \in A$.

В силу (1) для любых $y, z \in A$ выполняется равенство

$$
R(y, z)=R(z, y)
$$

В дальнейшем, коммутативность системы $A$ и равенство $(2)$ будем использовать без упоминания.

Пусть $q \in \Phi, q \neq 0$. Если введем на пространстве $E$ новое умножение

$$
X \circ Y=q(X Y+Y X)
$$

для любых $X, Y \in E$, то легко видеть, что алгебра $E^{\langle+\rangle}$относительно этого умножения является йордановой алгеброй. Через $R^{\langle+\rangle}(A)$ обозначим подалгебру алгебры $E^{\langle+\rangle}$, порожденную (относительно операции $X \circ Y$ ) всеми правыми умножениями системы $A$.

Далее, если не оговорено противное, будем предполагать, что $\Phi$ - поле характеристики $p \neq 2,3, q-$ фиксированной ненулевой элемент поля $\Phi$.

Пусть $\mathfrak{N}$ - подалгебра $E^{\langle+\rangle}$, содержащая $R^{\langle+\rangle}(A), B=A \dot{+} \mathfrak{N}$ - прямая сумма пространств $A$ и $\mathfrak{N}$ над $\Phi$. Определим на $B$ умножение $(\cdot)$, положив для любьх $x, y \in A$, $X, Y \in \mathfrak{N}$

$$
(x+X) \cdot(y+Y)=x Y+y X+\frac{1}{3} R(x, y)+X \circ Y .
$$

Относительно операции ( · ) пространство $B$ становится коммутативной алгеброй над $\Phi$ такой, что

$$
A \cdot A \subseteq \mathfrak{N}, \quad A \cdot \mathfrak{N} \subseteq A, \quad \mathfrak{N} \cdot A \subseteq A, \quad \mathfrak{N} \cdot \mathfrak{N} \subseteq \mathfrak{N}
$$

Равенство (3) равносильно равенствам

$$
x \cdot y=\frac{1}{3} R(x, y), \quad x \cdot Y=Y \cdot x=x Y, \quad X \cdot Y=X \circ Y
$$


Алгебру $B=A \dot{+} \mathfrak{N}$ назовем обертыв вающей q-алгеброй системы $A$. Если $\mathfrak{N}=R^{\langle+\rangle}(A)$, то обертывающую $q$-алгебру назовем стандартной.

В силу (4) для любых $x, y \in A$ имеем равенство $R(x, y)=3 x \cdot y$. Отсюда и из определения алгебры $R^{\langle+\rangle}(A)$ следует, что стандартная обертьвающая $q$-алгебра $B$ системы $A$ порождается всеми элементами системы $A$.

На линейном пространстве произвольной коммутативной алгебры $C$ над $\Phi$ введем операцию $\{x, y, z\}$, положив $\{x, y, z\}=J(x, y, z)$, где $J(x, y, z)=(x y) z+(z x) y+(y z) x-$ якобиан элементов $x, y, z$. Эта операция трилинейна и задает на $C$ коммутативную тройную систему, которую назовем ассоциированной и обозначим через $L(C)$.

Доказательства следуюших предложения, лемм 1 и 2 , аналогичные доказательствам соответствующих утверждений из [1], мы приведем в целях замкнутости изложения.

ПРЕДЛОЖЕНИЕ. Если $\Phi$ - поле характеристики $p \neq 3 u q \in \Phi$, то всякая коммутативная тройная система над $\Phi$ является подсистемой ассоииированной коммутативной тройной системы $L(B)$ любой своей обертывающей $q$-алгебры $B$.

ДоказАтельство. В силу (4) и коммутативности системы $A$ для любых $x, y, z \in A$ в $B$ имеем равенство

$$
\begin{aligned}
J(x, y, z) & =(x \cdot y) \cdot z+(z \cdot x) \cdot y+(y \cdot z) \cdot x \\
& =\frac{1}{3}(R(x, y) \cdot z+R(z, x) \cdot y+R(y, z) \cdot x) \\
& =\frac{1}{3}([z, x, y]+[y, z, x]+[x, y, z])=[x, y, z] .
\end{aligned}
$$

Следовательно, на $A$ операция $[x, y, z]$ совпадает с операцией $\{x, y, z\}$.

Предложение доказано.

Пусть $B=A \dot{+} R^{\langle+\rangle}(A)$ - стандартная обертывающая $q$-алгебра системы $A$. Если $I$ - идеал системы $A$, то через $R(I, A)$ обозначим идеал алгебры $R^{\langle+\rangle}(A)$, порожденный всеми правыми умножениями вида $R(i, x)$, где $i \in I, x \in A$ (относительно операции $X \circ Y=q(X Y+Y X))$.

Лемма 1. Если $I$ - идеал системы $A$, то $C=I \dot{+} R(I, A)$ - идеал ее стандартной обертывающей q-алгебры $B=A \dot{+} R^{\langle+\rangle}(A)$.

ДоКАЗАТЕЛЬСтво. В силу (4) и того, что $I \triangleleft A$, для любых $i \in I, x \in A, W \in R(I, A)$, $X \in R^{\langle+\rangle}(A)$ имеем

$$
\begin{aligned}
i \cdot x & =\frac{1}{3} R(i, x) \in R(I, A), \quad i \cdot X=i X \in I, \\
W \cdot x & =x W \in I, \quad W \cdot X=W \circ X \in R(I, A) .
\end{aligned}
$$

Отсюда и из коммутативности $B$ следует включение $C \cdot B \subseteq C$, т.е. $C \triangleleft B$. Лемма доказана.

Лемма 2. Если I - идеал стандартной обертывающей q-алгебры $B$ системы $A$, то его проекиия $I_{A}$ на пространство $А$ является идеалом системы $A$. 
ДокАЗАТЕЛЬСТво. Очевидно, что $I_{A}-$ подпространство $A$. Поэтому достаточно доказать, что $[v, x, y] \in I_{A}$ для любых $v \in I_{A}, x, y \in A$. Так как $v \in I_{A}$, то существует $V \in R^{\langle+\rangle}(A)$ такой, что $c=v+V \in I$. Положим $w=[v, x, y], W=V \cdot R(x, y)$. В силу (4)

$$
\begin{aligned}
w+W & =[v, x, y]+V \cdot R(x, y)=v \cdot R(x, y)+V \cdot R(x, y) \\
& =(v+V) \cdot R(x, y)=c \cdot R(x, y)=3 c \cdot(x \cdot y)
\end{aligned}
$$

Поскольку $c \in I$, то $c \cdot(x \cdot y) \in I$ и, следовательно, из последнего равенства следует включение $w+W \in I$, и так как $w \in A, W \in R^{\langle+\rangle}(A)$, то $w=[v, x, y] \in I_{A},\left[I_{A}, A, A\right] \subseteq I_{A}$, т.e. $I_{A} \triangleleft A$.

Лемма доказана.

ЛЕмма 3. Если $B$ - стандартная обертывающая q-алгебра системы $A$, то отобрахсение $\sigma$ алгебры $B$ в себя такое, что $\sigma(a)=-x+X$ для любого $a=x+X \in B$, где $x \in A, X \in R^{\langle+\rangle}(A)$, является инволютивным автоморфизмом алгебры $B$.

ДокАЗАТЕЛЬСТво. Очевидно, что отображение $\sigma$ взаимно однозначно, линейно и удовлетворяет равенству $\sigma^{2}=\varepsilon$, где $\varepsilon$ - тождественное отображение. Поэтому для доказательства леммы достаточно проверить, что $\sigma(a \cdot b)=\sigma(a) \cdot \sigma(b)$ для любых $a, b \in B$, $a=x+X, b=y+Y$, где $x, y \in A, X, Y \in R^{\langle+\rangle}(A)$. В силу (4) $x \cdot Y+X \cdot y \in A$, $x \cdot y+X \cdot Y \in R^{\langle+\rangle}(A)$. Поэтому по определению $\sigma$ и (4)

$$
\begin{aligned}
\sigma(a \cdot b) & =\sigma[(x+X) \cdot(y+Y)]=\sigma(x \cdot Y+X \cdot y)+\sigma(x \cdot y+X \cdot Y) \\
& =-(x \cdot Y+X \cdot y)+x \cdot y+X \cdot Y=-x \cdot(-y+Y)+X \cdot(-y+Y) \\
& =(-x+X) \cdot(-y+Y)=\sigma(a) \cdot \sigma(b) .
\end{aligned}
$$

Следовательно, $\sigma$ - инволютивньй автоморфизм алгебры $A$.

Лемма доказана.

Лемма 4. Если система $A$ проста, I - ненулевой идеал ее стандартной обертывающей q-алгебры $B$, то

$$
\begin{aligned}
I_{A} & =A, \\
I_{R^{\langle+\rangle}(A)} & =R^{\langle+\rangle}(A) .
\end{aligned}
$$

ДокАЗАТЕЛЬСТво. По лемме $2 I_{A}$ - идеал системы $A$ и, следовательно, в силу простоты $A$ либо $I_{A}=0$, либо $I_{A}=A$.

Если $I_{A}=0$, то $I \subseteq R^{\langle+\rangle}(A)$ и в силу (4) $A \cdot I \subseteq A \cdot R^{\langle+\rangle}(A) \subseteq A$. Следовательно, $A I=A \cdot I \subseteq A \cap I \subseteq I_{A}=0$. Поэтому элементы $I$ действуют тривиально на $A$, т.е. являются нулевыми эндоморфизмами пространства $A$. Следовательно, $I=0$, что противоречит условию леммы. Поэтому выполняется равенство (5).

Легко видеть, что $I_{R^{\langle+\rangle}(A)}$ - подпространство $R^{\langle+\rangle}(A)$. Докажем, что для любых $x, y \in A$

$$
R(x, y) \in I_{R^{\langle+\rangle}(A)}
$$


Действительно в силу (5) для любого $y \in A$ существует $Y \in R^{\langle+\rangle}(A)$ такой, что $d=y+Y \in I$. Но тогда в силу (4) для любого $x \in A$ имеем

$$
3 x Y+R(x, y)=3 x \cdot y+3 x \cdot Y=3 x \cdot(y+Y) \in I .
$$

Отсюда следует включение (7).

Если $X, Y$ - произвольные элементы из $I_{R^{\langle+\rangle}(A)}$, то существуют $x, y \in A$ такие, что $x+X, y+Y \in I$. Отсюда в силу (3)

$$
(x Y+y X)+\left(\frac{1}{3} R(x, y)+X \cdot Y\right)=(x+X) \cdot(y+Y) \in I .
$$

Поскольку $x Y+y X \in A, \frac{1}{3} R(x, y)+X \cdot Y \in R^{\langle+\rangle}(A)$, то из (8) следует включение

$$
\frac{1}{3} R(x, y)+X \cdot Y \in I_{R^{\langle+\rangle}(A)} .
$$

Так как $I_{R^{\langle+\rangle}(A)}$-подпространство пространства $R^{\langle+\rangle}(A)$, то из (7) и $(9)$ следует включение $X \cdot Y \in I_{R^{\langle+\rangle}(A)}$. Следовательно, $I_{R^{\langle+\rangle}(A)}$-подалгебра алгебры $R^{\langle+\rangle}(A)$. Но поскольку в силу $(7) I_{R^{\langle+\rangle}(A)}$ содержит все правые умножения $R(x, y)$, которые являются порождающими алгебры $R^{\langle+\rangle}(A)$, то вьполняется равенство (6).

Лемма доказана.

Теорема. Пусть $A$ - коммутативная тройная система над полем $\Phi$ характеристики $p \neq 2,3, B$ - ее стандартная обертывающая $q$-алгебра, где $q$ - ненулевой әлемент поля $\Phi$. Система $A$ проста, если проста алгебра В. Если система $A$ проста, mo

1) либо алгебра В проста;

2) либо $B=I_{1} \oplus I_{2}-$ прямая сумма (как идеалов) простых изоморфных алгебр $I_{1} u I_{2}$.

ДоказАТЕльство. Пусть стандартная обертьвающая $q$-алгебра $B$ системы $A$ проста. Если $I$ - произвольный ненулевой идеал системы $A$, то по лемме $1 C=I \dot{+} R(I, A)-$ идеал алгебры $B$ и в силу простоты $B$ имеем равенство $C=B$. Но тогда $I=A$ и, следовательно, система $A$ также проста.

Пусть система $A$ проста. Если алгебра $B$ проста, то доказьвать нечего. Если $B$ не является простой, то найдется ненулевой идеал $I$ алгебры $B$ такой, что $B \neq I$. Рассмотрим инволютивньй автоморфизм $\sigma$ алгебры $B$, определенный в формулировке леммы 3 . Образ $\sigma(I)$ идеала $I$ также является идеалом алгебры $B$. Следовательно, пересечение $C=I \cap \sigma(I)$ - идеал $B$.

Докажем, что $C=0$. Очевидно, что $\sigma(C) \subseteq C$. Поэтому по определению отображения $\sigma$ для любого $c=x+X \in C$ имеем $\sigma(c)=-x+X \in C$. Отсюда $x=\frac{1}{2}(c-\sigma(c)) \in C$, т.е. $C_{A} \subseteq C$. Предположим, что $C \neq 0$. Тогда по лемме $4 C_{A}=A$. Следовательно, $A \subseteq C$. Так как алгебра $B$ порождается элементами $A$, из последнего включения имеем включение $B \subseteq C$. Отсюда и из включения $C \subseteq I$ следует равенство $B=I$, что противоречит выбору идеала $I$. Следовательно, $C=0$. 
Докажем, что $B=I \oplus \sigma(I)$. В силу (5) для любого $x \in A$ существует $X \in R^{\langle+\rangle}(A)$ такой, что $a=x+X \in I$. Но тогда $\sigma(a)=-x+X \in \sigma(I)$. Следовательно,

$$
x=\frac{1}{2}(a-\sigma(a)) \in I+\sigma(I), \quad A \subseteq I+\sigma(I) .
$$

Аналогично, в силу (6) для любого $Y \in R^{\langle+\rangle}(A)$ существует $y \in A$ такой, что $b=$ $y+Y \in I$. Тогда

$$
Y=\frac{1}{2}(b+\sigma(b)) \in I+\sigma(I), \quad R^{\langle+\rangle}(A) \subseteq I+\sigma(I)
$$

Следовательно, $B=A \dot{+} R^{\langle+\rangle}(A)=I+\sigma(I)$ и поскольку $I \cap \sigma(I)=0$, то $B=I \oplus \sigma(I)$.

Пусть теперь $K$ - произвольньй ненулевой идеал $I$. Так как $B=I \oplus \sigma(I)$, то $K-$ идеал алгебры $B$. Следовательно, $B=K \oplus \sigma(K)$ и поскольку $K \subseteq I$, то $\sigma(K) \subseteq \sigma(I)$. Но тогда любой элемент $i \in I$ представляется в виде $i=k_{1}+\sigma\left(k_{2}\right)$, где $k_{1}, k_{2} \in K$. Отсюда и из включений $K \subseteq I, \sigma(K) \subseteq \sigma(I)$ имеем $i-k_{1}=\sigma\left(k_{2}\right) \in I \cap \sigma(I)=0$. Следовательно, $i=k_{1} \in K, I \subseteq K$. Поэтому $I=K, I$ - простая алгебра. Положив $I=I_{1}, \sigma(I)=I_{2}$, получим разложение $B=I_{1} \oplus I_{2}$.

Теорема доказана.

В заключение рассмотрим примеры стандартных обертывающих $q$-алгебр простых коммутативных тройных систем для наиболее интересного случая $q=1 / 2$.

ПримеР 1. Пусть $A_{1}-1$-мерная коммутативная тройная система над полем $\Phi$ характеристики $p \neq 2,3$ с базисньм элементом $e$ и умножением $[e, e, e]=3 e, B_{1}-$ ее стандартная обертывающая $\frac{1}{2}$-алгебра. Нетрудно проверить, что алгебра $R^{\langle+\rangle}\left(A_{1}\right)$ также 1 -мерна, и если $f_{11}=R(e, e)$ - базисный элемент $R^{\langle+\rangle}\left(A_{1}\right)$, то $f_{11} \circ f_{11}=3 f_{11}$. Алгебра $B_{1}=A_{1} \dot{+} R^{\langle+\rangle}\left(A_{1}\right)$ является 2 -мерной коммутативной алгеброй с базисом $e, f_{11}$ и таблицей умножения

$$
e \cdot e=\frac{1}{3} f_{11}, \quad e \cdot f_{11}=f_{11} \cdot e=3 e, \quad f_{11} \cdot f_{11}=3 f_{11}
$$

Легко видеть, что 1-мерные подпространства $I_{1}=\left\langle-3 e+f_{11}\right\rangle$ и $I_{2}=\left\langle 3 e+f_{11}\right\rangle$ являются идеалами алгебры $B_{1}, I_{1} \cap I_{2}=0$ и $B_{1}=I_{1} \oplus I_{2}$.

ЗАмЕчАниЕ. Нетрудно показать, что если $q \neq 1 / 2$, то стандартная обертьвающая $q$-алгебра системы $A_{1}$ проста.

ПримеР 2. Пусть $A_{2}-2$-мерная коммутативная тройная система над полем $\Phi$ характеристики $p \neq 2,3$ с базисом $e_{1}, e_{2}$ и таблицей умножения базисных элементов (c учетом коммутативности)

$$
\left[e_{1}, e_{1}, e_{1}\right]=0, \quad\left[e_{1}, e_{1}, e_{2}\right]=e_{2}, \quad\left[e_{1}, e_{2}, e_{2}\right]=e_{1}, \quad\left[e_{2}, e_{2}, e_{2}\right]=0
$$

Легко показать, что система $A_{2}$ проста. Положим

$$
f_{11}=R\left(e_{1}, e_{1}\right), \quad f_{12}=R\left(e_{1}, e_{2}\right), \quad f_{22}=R\left(e_{2}, e_{2}\right)
$$


Непосредственно проверяется, что $f_{11}, f_{12}, f_{22}$ линейно независимы и

$$
f_{11} \circ f_{11}=f_{11}, \quad f_{11} \circ f_{12}=\frac{1}{2} f_{12}, \quad f_{22} \circ f_{22}=f_{22} \text {. }
$$

Отсюда следует, что $\operatorname{dim} R^{\langle+\rangle}\left(A_{2}\right)=3$, т.е. стандартная обертьвающая $\frac{1}{2}$-алгебра $B_{2}$ является 5 -мерной коммутативной алгеброй. Алгебра $B_{2}$ имеет базис $e_{1}, e_{2}, f_{11}, f_{12}, f_{22}$ и таблицу умножения (с учетом коммутативности)

$$
\begin{aligned}
& e_{1} \cdot f_{11}=0, \quad e_{1} \cdot f_{12}=e_{2}, \quad e_{1} \cdot f_{22}=e_{1}, \\
& e_{2} \cdot f_{11}=e_{2}, \quad e_{2} \cdot f_{12}=e_{1}, \quad e_{2} \cdot f_{22}=0, \\
& e_{1} \cdot e_{1}=\frac{1}{3} f_{11}, \quad e_{1} \cdot e_{2}=\frac{1}{3} f_{12}, \quad e_{2} \cdot e_{2}=\frac{1}{3} f_{22}, \\
& f_{11} \cdot f_{11}=f_{11}, \quad f_{11} \cdot f_{12}=\frac{1}{2} f_{12}, \quad f_{12} \cdot f_{22}=\frac{1}{2} f_{12}, \quad f_{22} \cdot f_{22}=f_{22} \text {. }
\end{aligned}
$$

Поскольку размерность алгебры $B_{2}$ нечетна, то $B_{2}$ не может разлагаться в прямую сумму двух изоморфных подалгебр и, следовательно, проста.

\section{СПИСОК ЦИТИРОВАННОЙ ЛИТЕРАТУРЫ}

[1] Филиппов В. Т. Обертьвающие тройных систем и алгебр // Алгебра и логика. 1990. Т. 29. №1. C. $67-81$.

[2] ЖКевлаков К. А., Слинько А. М., Шестаков И. П., Ширшов А. И. Кольца, близкие к ассоциативным. М.: Наука, 1978.

[3] Lister W. G. A structure theory of Lie triple systems // Trans. Amer. Math. Soc. 1952. V. 72. №2. P. 217-242.

Институт математики СО РАН, г. Новосибирск 